\title{
Análisis económico de indicadores en el sector turístico en Cuba 2017-2020
}

DOI: https://doi.org/10.33262/ap.v1i3.2

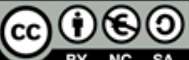

Economic analysis of indicators in the tourism sector in Cuba 2017-2020

\author{
Melissa Lemes Reyes ${ }^{1}$ \& Claudia Domínguez Cantera. ${ }^{2}$
}

\begin{abstract}
.
For Cuba's economy, tourism has been the driving force, this sector offers Cubans a large number of jobs and is also linked to many other sectors that are promoted by it. For this reason, the fallowing research aims to analyze the behavior of Cuba's tourism sector, mainly its main market sources during the 2017-2020 period, and stablishes indicators such as income, overnight stays and motives to travel among others, demonstrating their impact on the economy and the influence that the world have on them. For this research to be successful, mathematical and statistical methods are used from computer tools such as Microsoft Excel and SSPSS.
\end{abstract}

Keywords: Indicators, Overnight stays, Excel, SPSS.

\section{Resumen.}

Para la economía de Cuba el sector turístico ha sido su motor impulsor, pues esta esfera brinda al pueblo cubano gran cantidad de puestos de trabajo, además está vinculada con muchos otros sectores que son impulsados por este. Por ello la siguiente investigación tiene como objetivo analizar el comportamiento de la esfera turística de Cuba, fundamentalmente de sus principales mercados emisores durante el período del 2017-2020 y también se

\footnotetext{
${ }^{1}$ Universidad de La Habana. Facultad de Turismo. La Habana, Cuba. melylr@nauta.cu

${ }^{2}$ Universidad de La Habana. Facultad de Turismo. La Habana, Cuba. claudiacantera@nauta.cu
} 
establecerán indicadores como ingresos, pernoctaciones, motivos del viaje entre otros; para demostrar su incidencia en la economía y la influencia que tienen en ellos los fenómenos que suceden en el mundo. Para ello se emplean métodos matemáticos y estadísticos a partir de herramientas informáticas como el Microsoft Excel y el SPSS.

Palabras claves: Indicadores, Pernoctaciones, Excel, SPSS.

\section{Introducción.}

El turismo representa para todas las personas sin importar su procedencia una fuente de escape de la vida cotidiana, una brisa de aire fresco, una nueva forma de ver la vida, permite conocer nuevos lugares, diferentes culturas, transporta a otras épocas y donde las personas interpretan sus roles soñados. Cuba trabaja para ser uno de los principales destinos turísticos del Caribe, algo en lo que se ha convertido debido a su desarrollo en este sector año tras año.

Una de las ramas principales de la economía mundial es el turismo internacional. Numerosos países han priorizado el desarrollo del turismo. Uno de los indicadores fundamentales para evaluar su desempeño en los destinos receptores son los ingresos que genera esta actividad. El sector turístico interactúa con el resto de los sectores, convirtiendo al turismo en la locomotora de toda la economía, siendo fuente importante para la recuperación y expansión de una buena parte del sistema económico del país. El desarrollo turístico de Cuba siempre ha sido ascendente desde 1990 con unos 340 mil turistas. En 1996 alcanzó el primer millón, después pasaron 8 años para que llegara al segundo millón y luego 10 años para el tercer millón. En Cuba, como para la mayoría de los países del Caribe insular, los ingresos por turismo internacional se encuentran entre los primeros lugares como fuente de ingresos equivalente a exportaciones.

El turismo está sometido a altibajos en el crecimiento. Algunas de las caídas pueden atribuirse a problemas exógenos a la comercialización, a los que este sector es bien sensible, como las crisis económicas, naturales o político-sociales, incluidas las guerras sobre todo de carácter internacional.

Hoy en día son numerosos los indicadores que influyen en el desarrollo del turismo y en su crecimiento económico, principalmente en los años de 2017 a 2020, los cuales han tenido la incidencia de diferentes factores que han repercutido directamente en él. Por lo que se plantea el siguiente:

\section{Problema de investigación:}

¿Qué incidencia tienen algunos de los principales indicadores que influyen en el desarrollo del turismo en Cuba en su crecimiento económico en los años de 2017 a $2020 ?$

\section{Hipótesis:}


El análisis de algunos de los principales indicadores que inciden en el desarrollo turístico en Cuba durante los años de 2017 a 2020 permitirá conocer la incidencia de los mismos en el sector.

\section{Objetivo general:}

- Analizar la incidencia de algunos de los principales indicadores que inciden en el desarrollo económico del turismo durante los años de 2017 a 2020.

\section{Objetivos específicos:}

- Definir cuáles son algunos de los principales indicadores que inciden en el desarrollo económico del turismo durante los años de 2017 a 2020.

- Determinar los métodos y herramientas estadísticas necesarias para el análisis.

- Diagnosticar matemáticamente la incidencia de los indicadores en el desarrollo turístico.

\section{Metodología y herramientas:}

Para el cumplimiento de los objetivos trazados se emplearon como herramientas fundamentales el software informático Microsoft Excel y el estadístico SPSS.

Microsoft Excel: Desde la obtención de la información suministrada por el MINTUR y la ONEI se utilizó esta herramienta ya que los datos recopilados se encontraban en tablas de esos programas. Con esta herramienta se filtró la información necesaria para la investigación, se organizó en tablas y gráficos para un mejor aprovechamiento de la misma, se analizaron estadísticamente, se organizaron y los resultados obtenidos se utilizaron para la conformación del informe de investigación.

\section{Desarrollo del sector turístico durante el 2017.}

El 2017 fue un gran año para Cuba en materia de cifras del crecimiento turístico, que de no haber sido por el huracán Irma, que en septiembre atravesó el litoral norte del país afectando una gran parte de polos vacacionales que más visitantes reciben anualmente, lo que generalizó erróneamente una percepción en los viajeros de que todo estaba mal y que era imposible para Cuba recuperarse antes de la temporada alta; provocó menos visitas a la isla a pesar que desde el 1 de noviembre se reiniciaron las operaciones y servicios en la cayería norte. En el 2017, el crecimiento anual que rondaba los 100 mil o 200 mil visitantes, se elevó a más de 600 mil. Acumulando un total de 4653559 de arribos a Cuba.

El mercado canadiense mostró, en 2017, un leve decrecimiento, por los problemas asociados al debilitamiento de la tasa de cambio de la moneda canadiense en el mercado internacional; pero esto casi no se sintió en las estadísticas de la isla, las cuales estaban siendo impulsadas por el repunte de los visitantes estadounidenses y los cubanos residentes en ese país; además del favorable aporte de los tradicionales emisores como Francia, España e Italia, y en adición 
Rusia el cual desde agosto superó su cifra histórica, creciendo en un 68\%. También, nuevos mercados como Argentina y Brasil, implantaron récord ese año en las cifras de turistas hacia Cuba.

El turismo estadounidense alcanzó en 2017 un incremento asombroso, con respecto al 2016, del 191\% (casi el triple); lo cual se produce a pesar de las restricciones que impone el bloque de Estados Unidos a sus habitantes; y debido a que Cuba y EE.UU restablecieron relaciones en julio de 2015, entrando así en una etapa de normalización y de reanudación de vuelos comerciales, trayectos en cruceros y aprobación de medidas de flexibilización a los viajes.

La culminación de este año de manera tan satisfactoria de un total de 4653559 de arribos de visitantes, elevó la meta para el turismo del próximo año. Este resultado satisfactorio es reflejo de estrategias que se implementaron durante esos meses, como la recuperación de la modalidad de circuitos y los grupos de eventos, las nuevas operaciones aéreas en la temporada alta y la incidencia de la actividad de cruceros. Esta última modalidad, por ejemplo, reportó más de 397500 visitantes.

\section{Análisis del indicador arribos de visitantes a Cuba.}

Tabla 1: Total de arribos a Cuba desde el 2017-2019

\begin{tabular}{|c|c|c|c|c|c|c|c|c|c|}
\hline Países & $\begin{array}{l}\text { Total } \\
2017\end{array}$ & $\begin{array}{l}\text { Aéreo } \\
2018\end{array}$ & $\begin{array}{l}\text { Mar } \\
2018\end{array}$ & $\begin{array}{l}\text { Total } \\
2018\end{array}$ & $\begin{array}{c}\text { Aéreo } \\
2019\end{array}$ & $\begin{array}{l}\text { Mar } \\
2019\end{array}$ & $\begin{array}{l}\text { Total } \\
2019\end{array}$ & $\begin{array}{c}\text { Total } \\
\text { 2019-2018 }\end{array}$ & $\begin{array}{c}\text { Total } \\
2019 / 2018 \\
(\%)\end{array}$ \\
\hline Canadá & 1134225 & 1095291 & 14339 & 1109630 & 1112404 & 7673 & 1120077 & 10447 & 100,94 \\
\hline Com.Cubana & 546520 & 598158 & 2151 & 600309 & 623058 & 917 & 623975 & 23666 & 103,94 \\
\hline EE.UU & 619777 & 297360 & 341005 & 638365 & 298269 & 200269 & 498538 & -139827 & 78,10 \\
\hline Rusia & 105946 & 127557 & 9883 & 137440 & 174067 & 3910 & 177977 & 40537 & 129,49 \\
\hline Alemania & 243408 & 165858 & 42648 & 208506 & 154663 & 20293 & 174956 & -33550 & 83,91 \\
\hline Francia & 209642 & 171561 & 25960 & 197521 & 161246 & 8148 & 169394 & -28127 & 85,76 \\
\hline México & 142050 & 147799 & 24146 & 171945 & 160396 & 6599 & 166995 & -4950 & 97,12 \\
\hline España & 169507 & 139569 & 28400 & 167969 & 142416 & 3923 & 146339 & -21630 & 87,12 \\
\hline Italia & 228028 & 136020 & 41832 & 177852 & 122650 & 10786 & 133436 & -44416 & 75,03 \\
\hline Reino Unido & 205727 & 156412 & 33551 & 189963 & 103084 & 20661 & 123745 & -66218 & 65,14 \\
\hline Argentina & 99591 & 90262 & 7222 & 97484 & 92411 & 3167 & 95578 & -1906 & 98,04 \\
\hline China & 45899 & 43409 & 6535 & 49944 & 41362 & 3511 & 44873 & -5071 & 89,85 \\
\hline Turquía & 18894 & 15255 & 2301 & 17556 & 13456 & 1694 & 15150 & -2406 & 86,29 \\
\hline Otros & 884345 & 665144 & 299824 & 964967 & 641896 & 157767 & 784528 & -180439 & 77,57 \\
\hline Total general & 4653559 & 3834415 & 877498 & 4711913 & 3827935 & 447626 & 4275561 & -436352 & 90,7 \\
\hline
\end{tabular}

Fuente: elaboración propia a partir de los datos del MINTUR. 
Gráfico 1: total de arribos a Cuba.

\section{Total de arribos a Cuba.}

— Total 2017 - Total 2018 - Total 2019

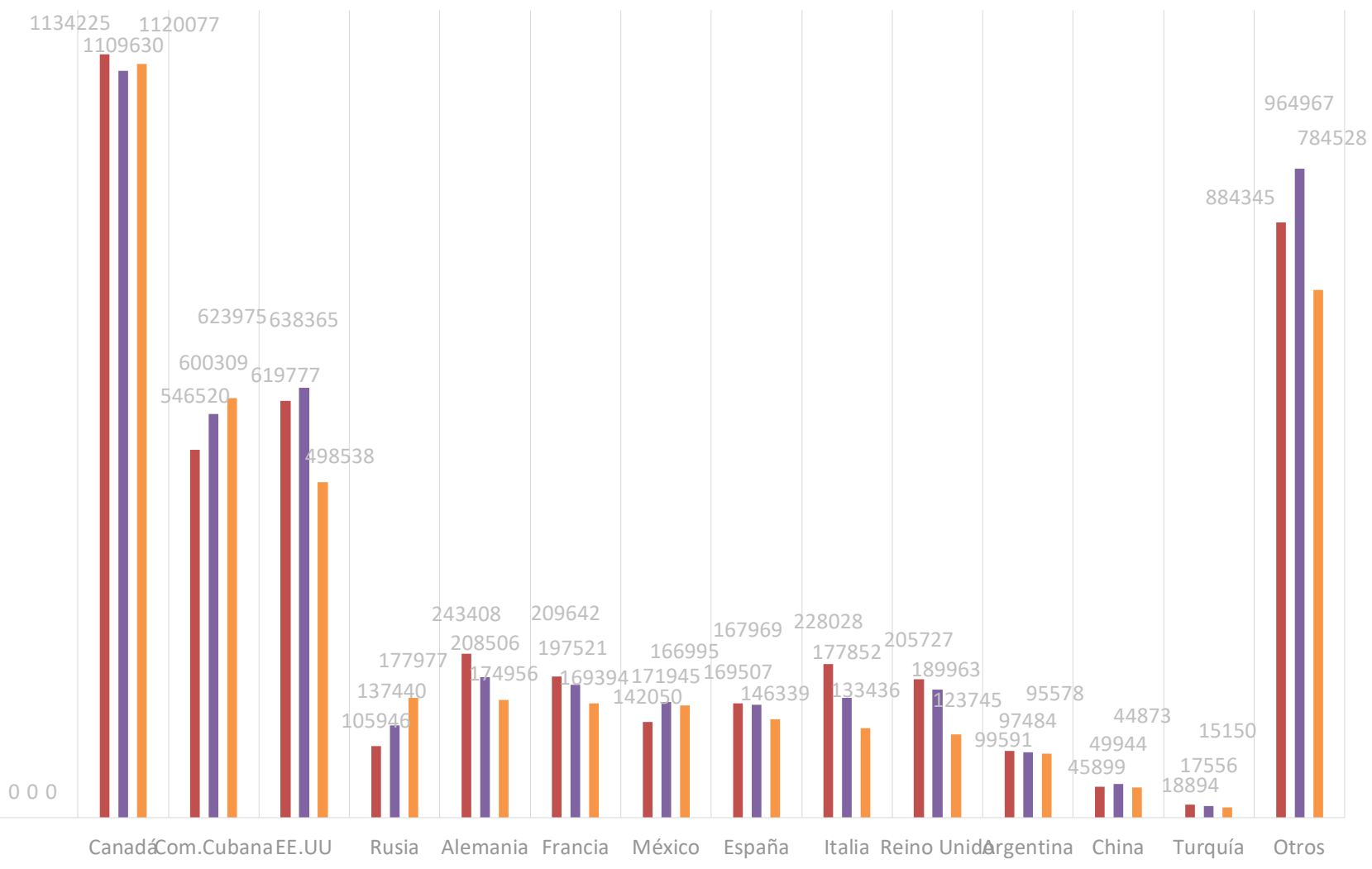

Fuente: Elaboración propia.

Durante el período del 2017-2019, se puede observar que el año que mayor cantidad de turistas arribaron a Cuba fue el 2018 con 4711913 turistas.

A pesar de que el país experimentó durante los primeros meses del año 2018 un importante descenso en el flujo de visitantes, ocasionado, principalmente por el paso de fenómenos naturales y por las políticas ejecutadas por la administración del presidente de los Estados Unidos, Donald Trump. La recuperación llegó a partir del periodo estival, temporada que se caracteriza por la llegada masiva de turistas de todo el mundo. El 2018 se cerró con un 42\% de repitencia, un índice bastante alto que demuestra la fidelidad de los que visitan la isla.

Estados Unidos, aunque muestra un crecimiento de un año a otro, debe su mayor peso a la cantidad de pasajeros de cruceros norteamericanos que se incluyen en el reporte de los visitantes, pues la cifra de norteamericanos que realizaron alguna pernoctación autorizada en Cuba retrocedió con respecto al año anterior (según datos del Ministerio del Turismo). 
Gráfico 2: Arribo de visitantes a Cuba.

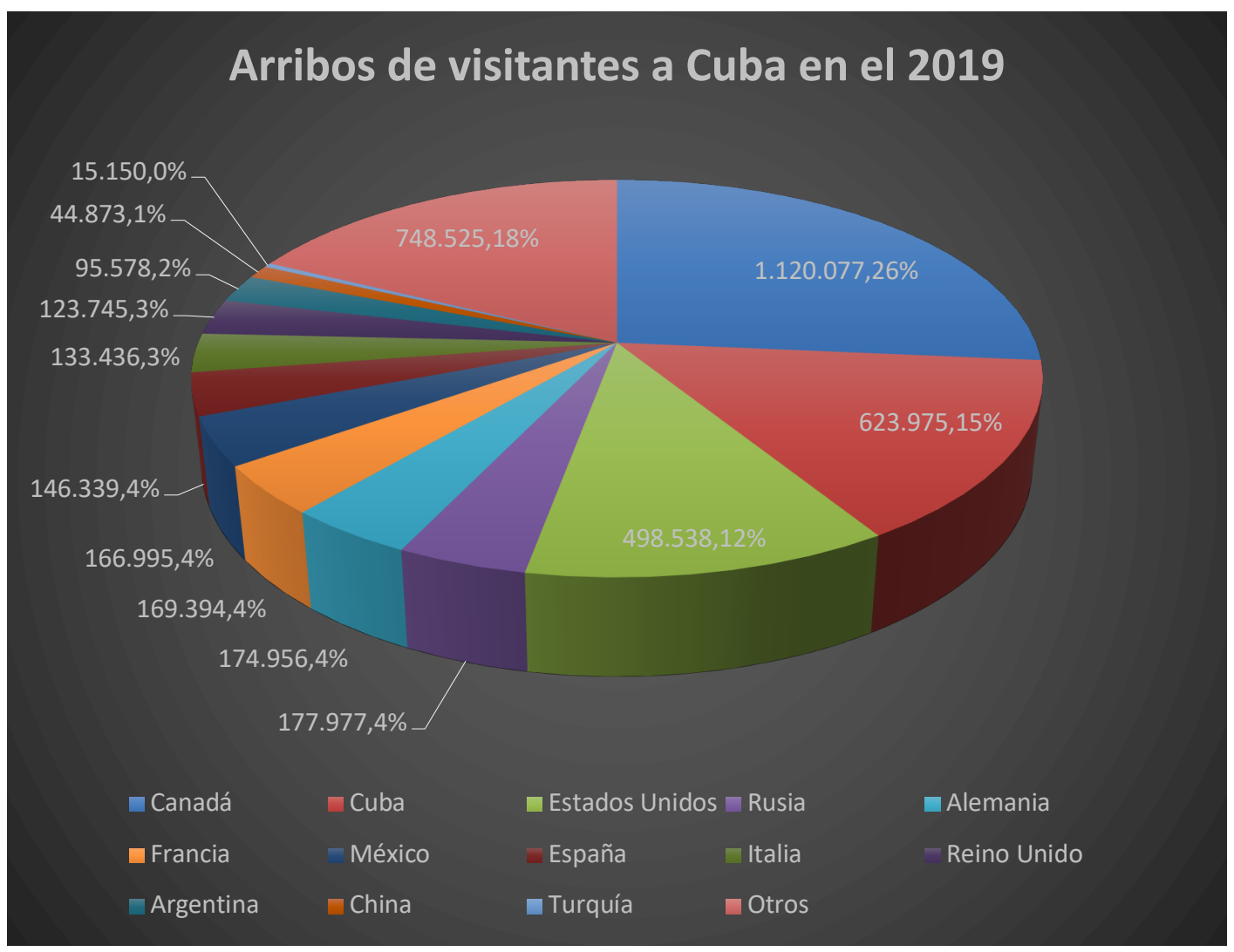

Fuente: Elaboración propia.

Canadá principal mercado emisor de turistas a Cuba.

Tabla 2: Llegada de turistas a Cuba.

\begin{tabular}{llllllll}
\hline & \multicolumn{2}{c}{ Canadá } & & & \multicolumn{3}{c}{$\begin{array}{c}\text { Total, de arribos de los } \\
\text { principales mercados }\end{array}$} \\
\hline Año & Mes & aire & mar & total & aire & mar & total \\
2017 & Enero & 160549 & 2240 & 162789 & 360189 & 36303 & 396492 \\
& Febrero & 160843 & 2125 & 162968 & 317390 & 27433 & 384828 \\
& marzo & 187382 & 2428 & 189810 & 418531 & 32432 & 280134 \\
& Abril & 141032 & 1325 & 142357 & 359217 & 23193 & 384696 \\
& Mayo & 68505 & 316 & 68821 & 258066 & 29393 & 287459 \\
& Junio & 53446 & 292 & 53738 & 243441 & 29345 & 272786 \\
& Julio & 71926 & 249 & 72175 & 289285 & 40107 & 329392 \\
& Agosto & 64435 & 393 & 64828 & 263945 & 42692 & 292732 \\
Septiembre & 17831 & 184 & 18015 & 118467 & 28298 & 146765 \\
& Octubre & 29080 & 380 & 29460 & 171567 & 36564 & 208131 \\
& Noviembre & 60215 & 880 & 61095 & 240490 & 36146 & 276636 \\
\hline
\end{tabular}




\begin{tabular}{llllllll}
\hline \hline \multirow{2}{*}{2018} & Diciembre & 106713 & 1456 & 108169 & 302571 & 44132 & 346703 \\
Enero & 146865 & 2177 & 149042 & 316479 & 42139 & 358618 \\
Febrero & 150001 & 2074 & 152075 & 305478 & 39356 & 360035 \\
marzo & 177118 & 1514 & 178632 & 390983 & 40936 & 431919 \\
Abril & 125282 & 1061 & 126343 & 289945 & 29610 & 339247 \\
Mayo & 62584 & 596 & 63180 & 231598 & 54733 & 286331 \\
Junio & 48078 & 599 & 48677 & 218524 & 52973 & 271497 \\
Julio & 56861 & 601 & 57462 & 253146 & 51182 & 304328 \\
Agosto & 52522 & 825 & 53347 & 234358 & 62525 & 296883 \\
Septiembre & 31091 & 596 & 31687 & 167702 & 49395 & 217097 \\
Octubre & 41828 & 1256 & 43084 & 183285 & 55676 & 235370 \\
Noviembre & 84673 & 1378 & 86051 & 237028 & 50113 & 306869 \\
Diciembre & 118388 & 1662 & 120050 & 311919 & 51335 & 363254 \\
Enero & 156846 & 2201 & 159047 & 328701 & 63338 & 353420 \\
Febrero & 155094 & 1395 & 156489 & 322608 & 47676 & 373284 \\
marzo & 179122 & 1611 & 180733 & 365110 & 52572 & 439795 \\
Abril & 126815 & 1470 & 128285 & 314244 & 58858 & 373102 \\
Mayo & 57110 & 883 & 57993 & 224687 & 57944 & 282631 \\
Junio & 43354 & 94 & 43448 & 215032 & 8612 & 223644 \\
Julio & 53186 & 1 & 53187 & 240496 & 297 & 240793 \\
Agosto & 48935 & 2 & 48937 & 224258 & 272 & 224530 \\
Septiembre & 30817 & 3 & 30820 & 161184 & 162 & 161346 \\
Octubre & 40456 & 3 & 40459 & 181046 & 527 & 167146 \\
Noviembre & 91476 & 4 & 91480 & 273730 & 680 & 179874 \\
Diciembre & 129193 & 6 & 129199 & 317252 & 613 & 317865 \\
\hline
\end{tabular}

Fuente: Elaboración propia.

De estos datos se tomó como variable independiente el total de turistas canadienses, y como variable dependiente el total de turistas de los principales mercados llegados en cada mes:

V1: Total de turistas canadienses. V2: Total de turistas de los principales mercados.

Con la aplicación de la regresión múltiple el objetivo es determinar la dependencia de estas variables.

El diagrama de dispersión es el primer método aplicado para demostrar la dependencia entre estas dos variables:

Este grafico demuestra la existencia de una correlación del tipo lineal positiva entre las variables estudiadas.

Grafico 3. Resultados del SPSS. 


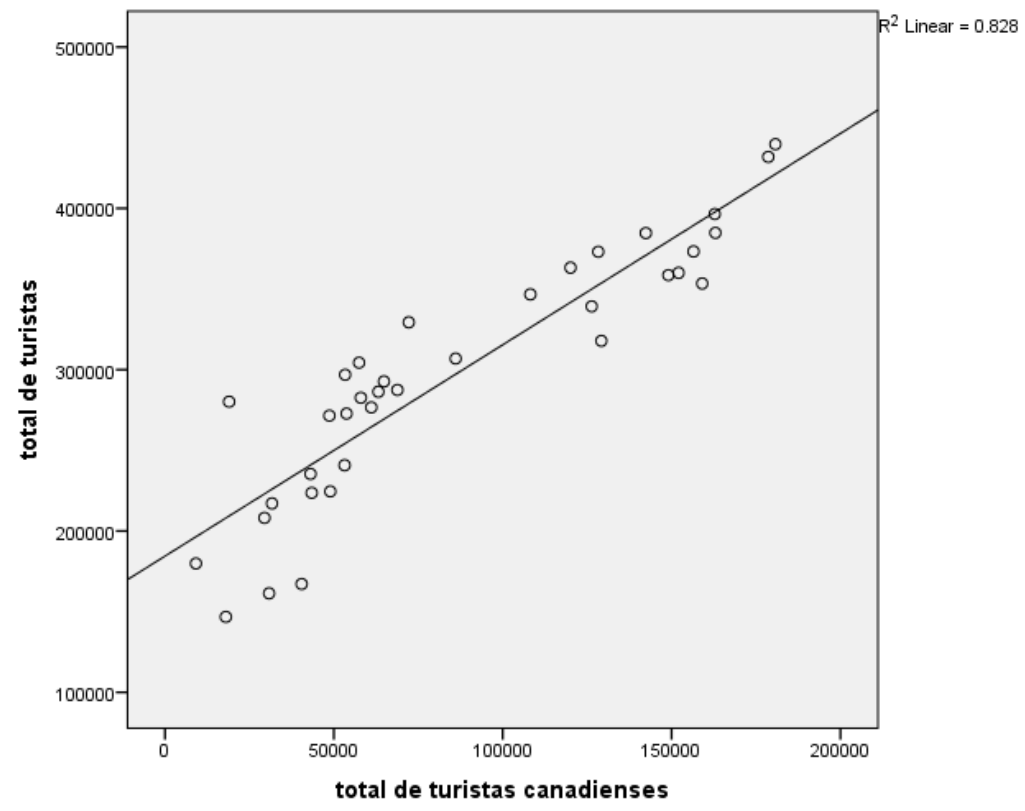

Fuente: Elaboración propia.

De igual manera el cálculo del coeficiente de correlación a partir del método de Pearson es de $\boldsymbol{\rho}=\mathbf{0 . 9 1}$, por lo que se puede afirmar que es una correlación muy alta, por estar en un intervalo entre 0.8 y 1 .

Analizando el diagrama de dispersión y las tablas 1 y 2 anteriores se puede observar que Canadá es el mercado emisor de turistas más grande que tiene Cuba y más constante; aumentando del 2018 al 2019 en un 0,94\% es decir aumento la cantidad de visitantes en 10447 representando Canadá el 26\% del total de turistas que arribaron a la isla en el 2019, pero esta cifra aún no supera a la del 2017 que fue de 1134225 visitantes.

La relación entre Cuba y Canadá están basadas en una larga historia de colaboración mutuamente beneficiosa y estrechos vínculos entre sus sectores que comenzó en el siglo XVIII y no fue hasta 1945 que instauraron relaciones diplomáticas. Actualmente Cuba representa el quinto destino receptor preferidos por la población canadiense, mientras para nosotros representa el principal mercado emisor.

\section{Análisis de otros mercados.}

Rusia viene creciendo desde el 2017 cuando rompió su cifra histórica y aumentó en el 2019 respecto al 2018 en un 29,49\%. Siendo el mercado que más creció y representó del total de turistas de ese año el 4\% aproximadamente. Lo cual lo ubica en el cuarto lugar emisor de extranjeros a la isla caribeña. Por todo esto Rusia será el invitado de honor en la venidera 
edición 40 de la feria de turismo FITCuba, al celebrarse en el 2020, el cual es el evento más importante del sector turístico.

Todo lo contrario, ocurrió con Estados Unidos que comparado con el 2018 tuvo 39827 turistas menos, lo que cayó en un 21,9\%. Representa de la cantidad total del 2019 el 12\%. Todo esto debido al aumento del bloqueo impuesto por el gobierno estadounidense.

El golpe más fuerte fue debido a la caída de turistas procedentes de Europa Occidental pues cinco (Francia, Alemania, Reino Unido, España e Italia) de los países que la conforman son importantes mercados para Cuba. Francia disminuyó su entrada de turistas en 28727, lo que significa que cayó en un 14,24\%. Alemania en 33550 extranjeros menos, lo que representa una caída $16,09 \%$. Italia en 44416 , lo que representa $24,97 \%$. España en 21630 , lo que es $12,88 \%$. Reino Unido en 66218 , lo que cayó en un34,86\%.

El decrecimiento combinado de estos cinco emisores equivale a 193941 viajeros menos es 1,38 veces mayor que la contracción de visitantes de EE. UU que fue de 139827 turistas menos.

Esta disminución tan garrafal en estos mercados, en la que resalta Reino Unido con un $34,86 \%$ está muy relacionada con la quiebra del gigante turoperador británico Thomas Cook tras 178 años de historia que cerró por no obtener un fondo de rescate lo que provocó la cancelación de reservaciones para sus más de 600000 vacacionistas en todo el mundo de ellos 2000 turistas que viajaron a Cuba, también la quiebra afectó a miles de personas que ya habían pagado sus reservas anticipadamente. Esto supone un duro golpe al turismo cubano, pues se pierde el principal puente para quienes visitaban la Isla desde el Reino Unido.

\section{Indicador: Cantidad de habitaciones turísticas en Cuba (2017-2018)}

Tabla 4: Habitaciones turísticas en Cuba (2017-2018).

\begin{tabular}{lrrr}
\hline \multicolumn{1}{c}{ Concepto } & $\mathbf{2 0 1 7}$ & $\mathbf{2 0 1 8}$ & CMA* \\
\hline $\begin{array}{l}\text { Habitaciones } \\
\text { entidades }\end{array}$ & 68.000 & 71.000 & $1,7 \%$ \\
$\begin{array}{l}\text { Alojamientos } \\
\text { privados }\end{array}$ & 22.000 & 24.000 & $27 \%$ \\
$\begin{array}{l}\text { Total } \\
\text { CMA*: Crecimiento } \\
\text { medio anual }\end{array}$ & 90.000 & 95.000 & $5,9 \%$ \\
\hline
\end{tabular}

Fuente: ONEI 2018.

A raíz del considerable crecimiento que ha experimentado el sector turístico en Cuba, se ha ampliado las capacidades de alojamiento turístico en esos años, en especial las habitaciones 
del sector estatal, aunque estas han sido menores que las llegadas de turistas, lo que impone una necesidad de seguir ampliando la planta hotelera estatal.

Gráfico 4: Habitaciones turísticas en Cuba (2017-2018).

\section{Habitaciones turísticas en Cuba}

$$
\text { - } 2017 \text { - } 2018
$$
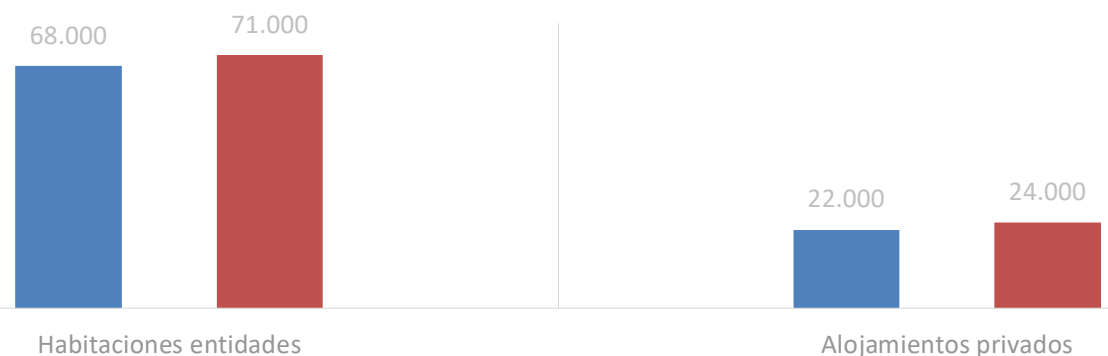

Fuente: Elaboración propia.

Indicador: Cantidad de cruceristas en Cuba (2017 y 2018)

Tabla 5: Cruceristas en Cuba (2017 y 2018).

\begin{tabular}{lrrr}
\hline \multicolumn{1}{c}{ Años } & $\mathbf{2 0 1 7}$ & $\mathbf{2 0 1 8}$ & Total \\
\hline $\begin{array}{l}\text { Cantidad } \\
\text { cruceristas }\end{array}$ & 586.710 & 850.000 & 1436710 \\
\hline
\end{tabular}

Fuente: Elaboración propia a partir de los datos del MINTUR 2018.

En 2017 se reportaban, ya en noviembre unos 397530 cruceristas, en 230 buques de diez compañías diferentes que tocaron puertos cubanos. Desde alternativas de ocio tan diferentes, y a partir de una estrategia de desarrollo cada vez mejor planeada, Cuba logró además en 2017 incrementar su cifra de turistas días y registrar una mejoría notable en indicadores relacionados con la calidad. (http://www.excelenciascuba.com/noticia/apuntes-de-interessobre-el-turismo-cubano-en-2017)

Cabe resaltar la actividad de los cruceros en la isla. En 2018 eran 17 las compañías de cruceros operando en el país. Según fuentes del Ministerio del Turismo, hasta el mes de noviembre de 2018 habían arribado 793 mil cruceristas (con un crecimiento de un 45\% respecto al año anterior).

En la tabla anterior se aprecia un incremento de la cifra de cruceristas de 2018 con respecto a 2017; todo esto influido por las estadísticas de una satisfacción general con la mayoría de los vacacionistas, expresando que recomendarían el destino a sus familiares y amigos, o manifestando su deseo de volver a visitarlo en próximos meses. 
Indicador: pernoctaciones de turistas internacionales en alojamientos por tipo de establecimiento.

Tabla 6: Pernoctaciones de turistas internacionales en el conjunto de los medios de alojamiento por tipo de establecimiento.

\begin{tabular}{|c|c|c|c|}
\hline Tipo de establecimiento & 2017 & 2018 & Promedio* \\
\hline Total & 33.085 .823 & 28.840 .669 & 30.963 .246 \\
\hline Hoteles y otros establecimientos & 21.515 .483 & 19.641 .711 & 20.578 .597 \\
\hline Hoteles & 21.412 .748 & 19.536 .118 & 20.474 .433 \\
\hline 5 Estrellas & 9.476 .455 & 8.634 .248 & 9.055 .352 \\
\hline 4 Estrellas & 7.954 .030 & 7.907 .035 & 7.930 .533 \\
\hline 3 Estrellas & 2.286 .048 & 1.969 .512 & 2.127 .780 \\
\hline 2 Estrellas & 1.671 .155 & 1.007 .939 & 1.339 .547 \\
\hline 1 Estrella & 25.060 & 17.384 & 21.222 \\
\hline Otros establecimientos & - & - & - \\
\hline Moteles & 29.999 & 30.550 & 30.275 \\
\hline Hoteles-Apartamentos ${ }^{\text {(a) }}$ & 46.731 & 49.013 & 47.872 \\
\hline Hostal & 26.005 & 26.030 & 26.018 \\
\hline Medios de alojamiento complementarios & 11.570 .340 & 9.198 .958 & 10.384 .649 \\
\hline Casas y cabañas (b) & 59.567 & 62.068 & 60.818 \\
\hline De ellos: Bases de campismo & 33.788 & 37.438 & 35.613 \\
\hline Campamentos & 3.025 & 3.135 & 3.080 \\
\hline Villas turísticas & 528.108 & 533.281 & 530.695 \\
\hline Otros medios de alojamientos & 10.979 .640 & 8.600 .474 & 9.790 .057 \\
\hline De ello: Sector privado & 10.972 .649 & 7.897 .851 & 9.435 .250 \\
\hline $\begin{array}{l}\text { (a) Se refiere a apartamentos turísticos } \\
\text { aparthoteles. } \\
\text { (b) Incluye Operación Milagro en el año } 2007\end{array}$ & & & \\
\hline
\end{tabular}

Fuente: Series de datos de la ONEI (Turismo) 2018. (*Elaboración propia).

Tal y como se muestra en la tabla, según los datos ofrecidos por la Oficina Nacional de Estadística hubo un decrecimiento de las pernoctaciones entre estos dos años, relacionado, entre otros factores por el aumento de los cruceros. También es apreciable el predominio de las pernoctaciones en los establecimientos del sector estatal.

Indicador: ingresos de las entidades turísticas en Cuba 2018-1019

Tabla 7: ingresos de las entidades turísticas en Cuba del 2018-2019 en miles de CUC.

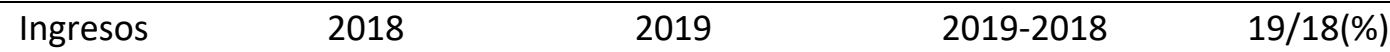


ISSN: 2773-7330

Vol. 1, No 3, p. 53-69

octubre-diciembre, 2019

\begin{tabular}{lllll}
\hline \hline total & 2191860,1 & 2184602,6 & $-7257,5$ & 99,7 \\
alojamiento & 622852,5 & 571098,7 & $-51753,8$ & 91,7 \\
$\begin{array}{l}\text { Comercio } \\
\text { minorista }\end{array}$ & 272162,4 & 274301,8 & 2139,4 & 100,8 \\
gastronomía & 857445,6 & 897816,5 & 40370,9 & 104,7 \\
transporte & 186768,5 & 177263,7 & $-9504,8$ & 94,9 \\
Recreación & 39324,2 & 38497,1 & $-827,1$ & 97,9 \\
Otros & 213306,9 & 225624,8 & 12317,9 & 105,8 \\
\hline
\end{tabular}

Fuente: Elaboración propia.

Gráfico 4: Ingresos en el 2019

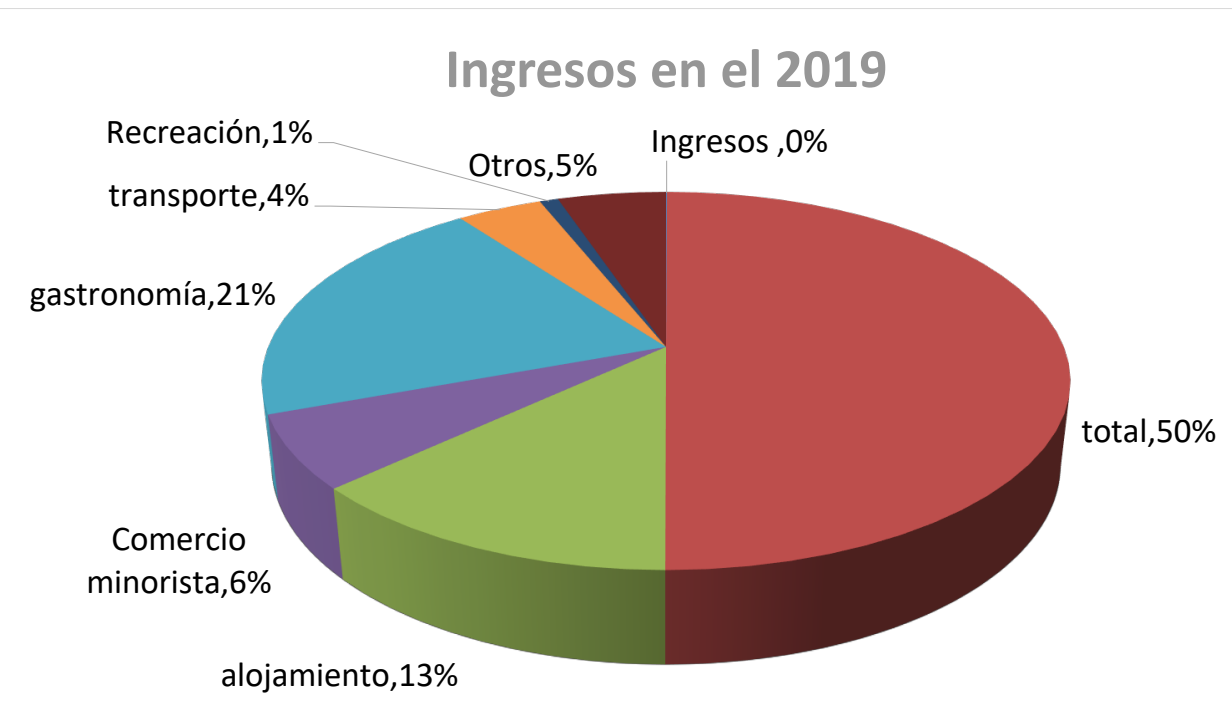

Fuente: elaboración propia.

Indicador: motivo de los viajes de los visitantes a Cuba del 2017-2019

Tabla 8: motivo de los viajes de los turistas del 2017-2019

\begin{tabular}{|c|c|c|c|c|c|c|c|}
\hline \multirow[t]{2}{*}{ Motivos } & 2017 & 2018 & (\%) & 2019 & \multirow{2}{*}{$\begin{array}{l}\%) \\
2019 \\
\end{array}$} & \multirow{2}{*}{$\begin{array}{l}2019- \\
2018\end{array}$} & \multirow{2}{*}{$\begin{array}{l}(\%) \\
19 / 18\end{array}$} \\
\hline & & & 2018 & & & & \\
\hline $\begin{array}{l}\text { Ocio, recreo y } \\
\text { vacaciones. }\end{array}$ & 4326035 & 4307239 & 91 & 3994822 & 93 & -312417 & 92,7 \\
\hline
\end{tabular}


ISSN: 2773-7330

www.alfapublicaciones.com octubre-diciembre, 2019

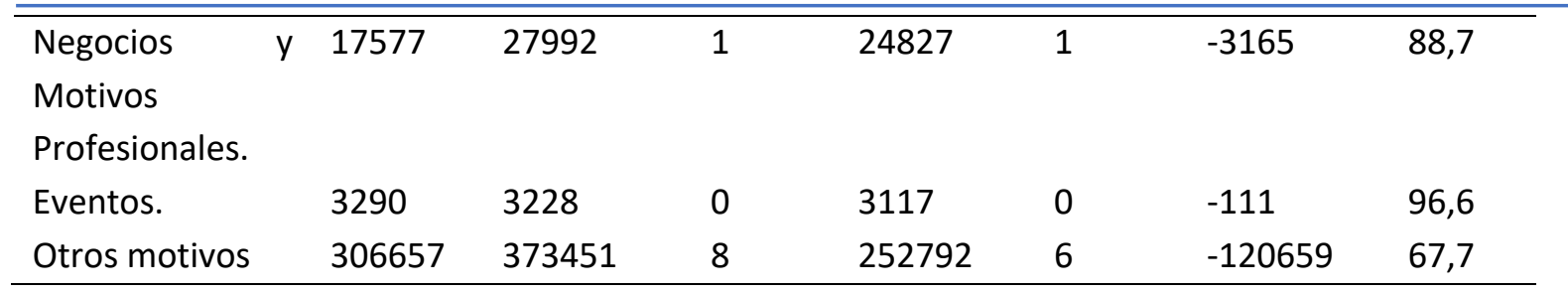

Fuente: Elaboración propia a partir de los datos de Turismo Internacional Seleccionados. Enero-diciembre 2019. ONEI

Tabla 9: Análisis de los motivos de viaje desde el 2012-2019

\begin{tabular}{lcc}
\hline Años & Ocio y vacaciones & Otros motivos \\
\hline 2012 & $94,7 \%$ & $5,2 \%$ \\
2013 & $95,1 \%$ & $4,8 \%$ \\
2014 & $94,6 \%$ & $5,4 \%$ \\
2015 & $94,5 \%$ & $5,5 \%$ \\
2016 & $93,5 \%$ & $6,5 \%$ \\
2017 & $92,8 \%$ & $7,2 \%$ \\
2018 & $91 \%$ & $9 \%$ \\
2019 & $93 \%$ & $7 \%$ \\
\hline
\end{tabular}

Fuente: Elaboración propia a partir de los datos reflejados en los anuarios de turismo publicados en ONEI.cu

En esta tabla demuestra que el principal motivo de la visita de los turistas a la isla es turismo de sol y playa (Ocio, recreo y vacaciones) pues Cuba cuenta con un excelente clima con temperaturas entre los 25 y los 30 grados centígrados; con playas espectaculares que poseen arenas finas y aguas cálidas y transparentes. Cerca de estas maravillas de paisajes se encuentran hermosos balnearios bien equipadas que brindas actividades de ocio y esparcimiento a sus huéspedes. Entre los destinos de playa se destaca por excelencia Varadero con aguas inolvidables color turqués y con barreras coralinas accesibles a simple nado, además brinda una amplia gama de hoteles 5 y 4 estrellas más de 50 guardan exclusivas playas privadas.

Indicador: edad y sexo de los visitantes en el 2019

Tabla 10: Llegada de visitantes por edades y sexos en el 2019. 


\begin{tabular}{crcc}
\hline Edades & Total & Masculino & Femenino \\
\hline & 4275558 & 2123626 & 2151932 \\
Menos de 15 & 306762 & 152832 & 153930 \\
De 15 a 24 & 351034 & 151019 & 200015 \\
De 25 a 44 & 1501257 & 747975 & 753282 \\
De 45 a 59 & 1211866 & 612400 & 599466 \\
De 60 y más & 904639 & 459400 & 445239 \\
\hline
\end{tabular}

Fuente: Oficina Nacional de Estadística e Información.

La diferencia entre la cantidad de visitantes de un sexo y de otro no son alarmantes, pues este factor no es determinante en el sector del turismo.

Gráfico 6: Cantidad de visitantes por edades.

\section{Cantidad de visitantes por edades.}

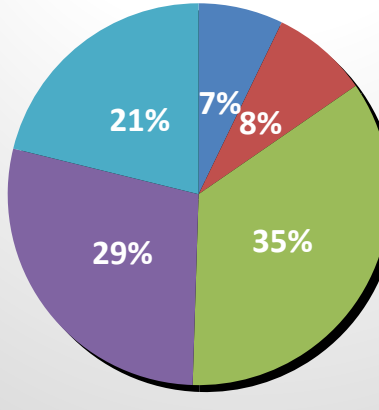

- Menos de 15

De 15 a 24

De 25 a 44

De 45 a 59

- De 60 y más

Fuente: Elaboración propia.

A partir de este gráfico se puede observar que la mayor cantidad de turistas que visitan a Cuba son de 25 a 44 años lo que demuestra que el país debe seguir motivando a este sector con ofertas tentadoras y espectaculares.

\section{El sector turístico en el 2020.}

El cierre del 2019 representó resultados negativos para el turismo pues hubo una disminución del 9,3\% en comparación con el año anterior.

En el mes de enero del 2020 visitaron el país 393762 visitantes, que significan 95856 menos que en el igual mes de 2019. 
ISSN: 2773-7330

Tabla 11: Llegada de los principales mercados emisores en el mes de enero a Cuba en el 2019 y 2020.

\begin{tabular}{lllll}
\hline Países & 2019 & 2020 & $2020-2019$ & $2020 / 2019(\%)$ \\
Visitantes & 489618 & 393762 & -95856 & 80,4 \\
Canadá & 159022 & 162307 & 3285 & 102,1 \\
Comunidad Cubana en & 42945 & 37394 & -5551 & 87,1 \\
el Exterior & 16742 & 24842 & 8100 & 148,4 \\
Rusia & 62416 & 19464 & -42952 & 31,2 \\
Estados Unidos & 22354 & 16871 & -5483 & 75,5 \\
Francia & 20891 & 14679 & -6212 & 70,3 \\
Alemania & 16522 & 13129 & -3393 & 79,5 \\
Italia & 10951 & 10580 & -371 & 96,6 \\
México & 9485 & 8910 & -575 & 93,9 \\
España & 15945 & 1989 & -13956 & 50,1 \\
Inglaterra & 112345 & 77597 & -34748 & 69,1 \\
Otros & & & & \\
\hline
\end{tabular}

Fuente: elaboración propia a partir de los datos de la ONEI. Arribos a Cuba en enero 2020.Desempeños de los principales mercados.

Estados Unidos tuvo una disminución evidente de un 68,8\%debido a las nuevas restricciones al turismo impuesta por su gobierno.

Canadá continuó siendo el principal emisor de turistas a la isla, seguido por la Comunidad Cubana en el Exterior y en tercero Rusia quien tuvo un aumento de un 48,4\%.

Inglaterra tuvo un descenso del 49,9\%, Italia de 20,5\%, España tuvo una disminución de $6,1 \%$, Alemania de $29,7 \%$ debido a lo ya mencionada quiebra de la turoperadora Thomas Cook la más importante para Cuba.

\section{Conclusiones.}

- Se identificó por primera vez en diciembre de 2019 una enfermedad por coronavirus iniciada en Wuhan, en la República Popular de China que azotará al mundo en el 
2020. Pues está gran pandemia, reconocida así en marzo es de rápido contagió y ha paralizado al mundo y con ella sus sectores económicos.

- Los 3 primeros casos confirmados de Covid-19 en Cuba fueron el 11 de marzo del 2020 los cuales corresponden a tres turistas de nacionalidad italiana, provenientes de la región de Lombardía.

- Cuba anunció el cierre de sus fronteras el 20 de marzo del 2020 excepto para los residentes en la isla. Esta medida fue tomada con el objetivo para evitar la llegada de nuevos casos de COVID-19 al país.

- A pesar de esto el sector turístico sigue preparándose para abrir sus fronteras más adelante para el mundo y la Feria Internacional del Turismo programada para mayo del 2020 fue pospuesta para más adelante.

\section{Referencias bibliográficas.}

Alina. (9 de enero de 2018). Excelencias Cuba. Obtenido de http://www.excelenciascuba.com/noticia/apuntes-de-interés-sobre-el-turismocubano-en-2017

Cabrera, D. J. (16 de enero de 2020). El economista de Cuba. Obtenido de http://www.eleconomista.cubaweb.cu/el-turismo-en-Cuba,-entre-sanciones-yprohibiciones

Castro, H. A. (enero-junio de 2020). Comportamiento de ingreos por turismo internacional en Cuba 2014-2018. SciELO. Economía y Desarrollo, 163(1).

Cuba anunció el cierre de sus fronteras al turismo por treinta días. (2020). infobae.

Cubadebate. (25 de enero de 2019). Obtenido de Arribo de visitantes internacionales a Cuba crece 10 porciento en lo que va de año: www.cubadebate.cu

Fernández, F. M. (2018). Turismo en Cuba: récord y expectativa-. Cubahora.

La quiebra de Thomas Cook afecta a unos 2000 turistas en Cuba. (2019). EFE.

Oficina Nacional de Estadística e Información. (Edición marzo 2020). Turismo Internacional Indicadores Seleccionados Enero-.

wikipedia. (2020). Pandemia de enfermedad por coronavirus de 2019-2020.

wikipedia. (2020). Pandemia de enfermedad por coronavirus de 2020 en Cuba.

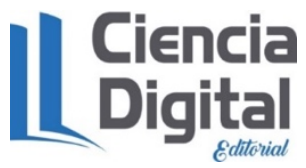




\section{Para citar el artículo indexado}

Lemes Reyes, M. (2020). Análisis económico de indicadores en el sector turístico en Cuba 2017-2020. AlfaPublicaciones, 1(3), 53-69. https://doi.org/10.33262/ap.v1i3.2

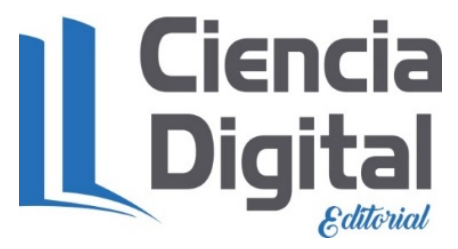

El artículo que se publica es de exclusiva responsabilidad de los autores y no necesariamente reflejan el pensamiento de la Revista Alpha Publicaciones.

El artículo queda en propiedad de la revista y, por tanto, su publicación parcial y/o total en otro medio tiene que ser autorizado por el director de la Revista Alpha Publicaciones.
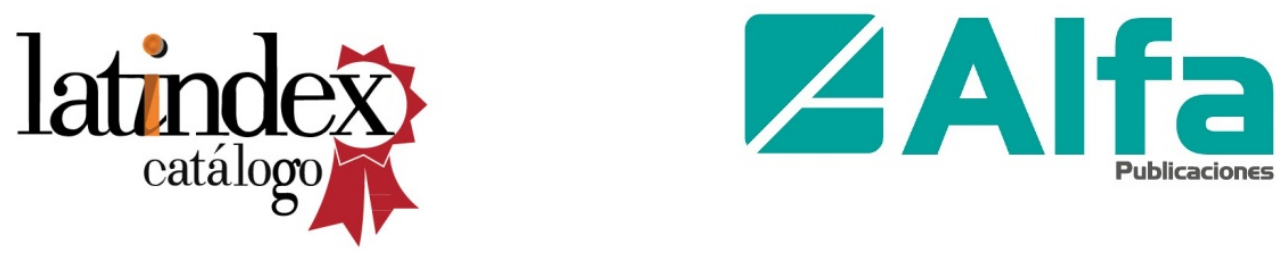